\title{
Convection dans les planètes « molles »: du laboratoire à Vénus
}

\author{
Anne Davaille $^{(1)}$ (davaille@fast.u-psud.fr), Erika Di Giuseppe ${ }^{(1)}$, Eric Mittelstaedt ${ }^{(2,1)}$ \\ et Suzanne Smrekar ${ }^{(3)}$ \\ (1) Laboratoire FAST (CNRS - Université Paris-Saclay), Centre universitaire, \\ 91405 Orsay Cedex \\ (2) Department of Geological Sciences, University of Idaho, Moscow, Idaho, USA \\ (3) Jet Propulsion Laboratory, NASA-CalTech, Pasadena, CA, USA
}

\section{Dans les manteaux des planètes}

rocheuses et dans les dispersions colloïdales, une variation

de température ou de composition peut induire des variations de densité, la gélification de leur microstructure et une modification drastique de leur viscosité.

\section{Mouvements de convection}

et formation d'une peau faiblement déformable en surface peuvent

\section{donc coexister.}

\section{L'étude de la convection}

dans les dispersions colloïdales peut fournir des informations précieuses sur la physique des planètes. Ainsi, nous avons montré que les panaches thermiques sont importants pour casser la peau de surface et induire la subduction de celle-ci, un phénomène qui pourrait être actif sur Vénus aujourd'hui.

\section{Convection dans les planètes}

La convection se développe lorsqu'un gradient instable de densité est présent dans une couche fluide placée dans un champ de gravité : le matériau plus dense coule et le plus léger remonte. Ce gradient de densité peut être induit par un gradient de température, lorsque la couche de fluide est chauffée par en dessous et refroidie par le dessus (la configuration classique de «Rayleigh-Bénard ", fig. 1). La convection est un processus majeur dans la dynamique des océans, de l'atmosphère, de l'intérieur des étoiles et des planètes.

En effet, formée chaude dans un univers froid, une planète voit son évolution dictée par son refroidissement, qui est lui-même contrôlé par la convection se développant dans son enveloppe la plus visqueuse. Dans une planète rocheuse comme la Terre, il s'agit du " manteau », c'est-à-dire de la couche de roches solides, épaisse de $2900 \mathrm{~km}$, située entre la croute terrestre et le noyau de fer liquide. On observe aujourd'hui des régimes convectifs radicalement différents dans la Terre et ses deux planètes « sœurs ", Vénus et Mars, bien qu'elles aient toutes trois des compositions similaires et des traces de volcanisme, signe de planètes encore "actives ». Ainsi, la surface de la Terre est découpée en " plaques » qui se déplacent latéralement à des vitesses de quelques centimètres par an, et coulissent les unes par rapport aux autres (la " tectonique des plaques »). L'intérieur des plaques est très peu déformé, la déformation étant fortement localisée à leurs frontières. $60 \%$ de ces plaques (la fraction située dans les océans) se renouvelle continument par "subduction ", lorsqu'elles replongent dans le manteau, et par « accrétion » le long des dorsales océaniques, où les nouvelles plaques se forment puis s'écartent (fig. 2A). En revanche, la

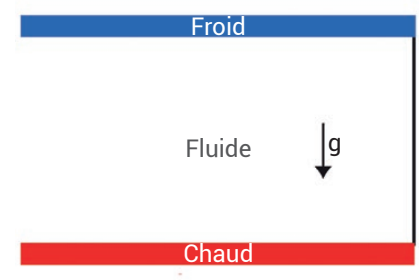

a

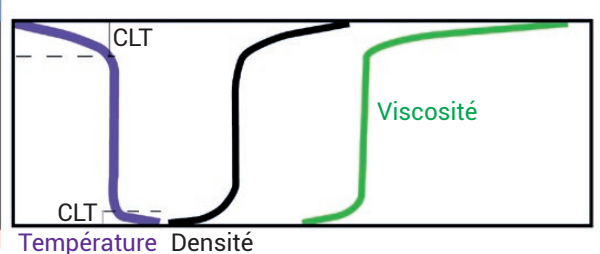

(b)

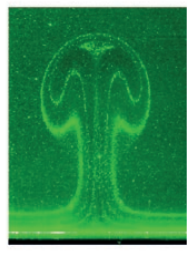

C

1. Convection thermique.

(a) Système chauffé par en dessous et refroidi par au-dessus.

(b) Profils verticaux correspondants de température, densité et viscosité. "CLT " désigne les couches limites thermiques du système. Sur une planète, la CLT supérieure correspond à la "plaque tectonique".

(c) Panache thermique chaud se développant pour des nombres de Rayleigh Ra $>10^{6}$ (défini plus loin); les lignes lumineuses correspondent à des isothermes. 


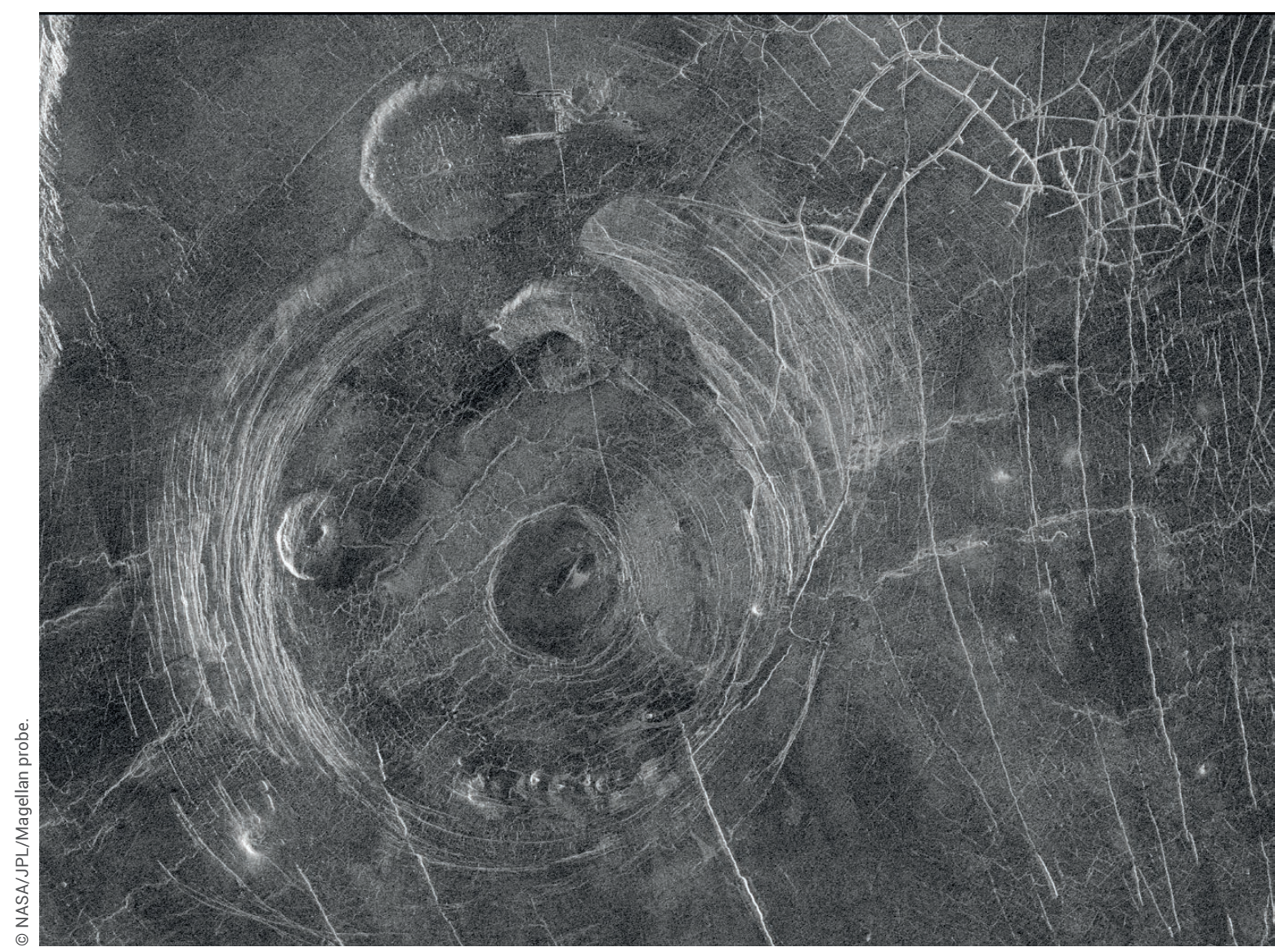

Image radar d'une corona (formation géologique circulaire) d'environ 200 km de diamètre sur Vénus, prise en 1991 par le satellite Magellan de la NASA. Cette corona est probablement due à l'impact d'un panache chaud qui serait remonté dans le manteau. Sur l'image, on observe également des dômes d'origine volcanique (à l'intérieur et au-dessus de la corona) et un réseau complexe de fractures (en haut, à droite).

surface de Mars est très ancienne et la tectonique des plaques s'y est probablement arrêtée il y a plus de 4 milliards d'années. Vénus présente un cas intermédiaire, avec la majorité de sa surface d'un âge estimé entre 150 et 750 millions d'années (Ma). Il a été proposé que la surface de Vénus se renouvelait épisodiquement au cours d'évènements catastrophiques, pendant lesquels elle se fracture et toutes les plaques replongent dans le manteau.

Les conditions de sélection d'un régime donné, et, en particulier, de l'initiation de la subduction, sont encore très débattues. La difficulté vient de la complexité de la rhéologie des roches, qui dépend de la température, de la pression, du taux de cisaillement, des contraintes appliquées, de l'endommagement et de la composition. La température a l'effet le plus important, les roches s'écoulant comme un fluide visqueux à haute température et sur de longues durées $(\sim 100 \mathrm{Ma})$, mais étant élastiques et cassantes comme un solide à faible température et sur les temps courts (par exemple dans les tremblements de Terre). Modéliser ce comportement et caractériser son influence sur la convection est l'un des défis actuels de la géodynamique.

\section{Le regard de la mécanique des fluides et de la physique de la matière " molle »}

Ce comportement, soit fluide soit solide selon les circonstances, est caractéristique de la matière " molle ". Certaines dispersions colloïdales aqueuses de nanoparticules de silice [1], telles que le Ludox, présentent une rhéologie analogue à celle des roches, allant de visqueuse à cassante lorsque la concentration en particules $\phi$ augmente et la fraction en solvant $(1-\phi)$ diminue. Cette dernière tient ainsi un rôle analogue à la température dans une planète. Nous avons donc étudié en laboratoire les différents régimes de convection lorsqu'une couche épaisse de Ludox est séchée par le dessus par évaporation à faible humidité et chauffée par en dessous. L'intensité de la convection dépend du nombre de Rayleigh $R a$, rapport de l'effet moteur de la convection (la force d'Archimède) sur les effets antagonistes (la diffusion de la chaleur ou de la concentration, et les forces visqueuses qui diffusent la quantité de mouvement) :

$R a=\Delta \rho g H^{3} /(\kappa \eta)$, où $g$ désigne l'accélération de la pesanteur, $H$ l'épaisseur de fluide, $\kappa$ la diffusivité thermique ou chimique, $\eta$ la viscosité dynamique du fluide, et $\Delta \rho$ le contraste de densité entre les couches limites. Dans le cas du séchage d'une solution colloïdale en laboratoire, le contraste de densité $\Delta \rho$, moteur de la convection, est dû aux variations de la concentration $\phi$ induites par l'évaporation. Pour un manteau planétaire, le contraste 


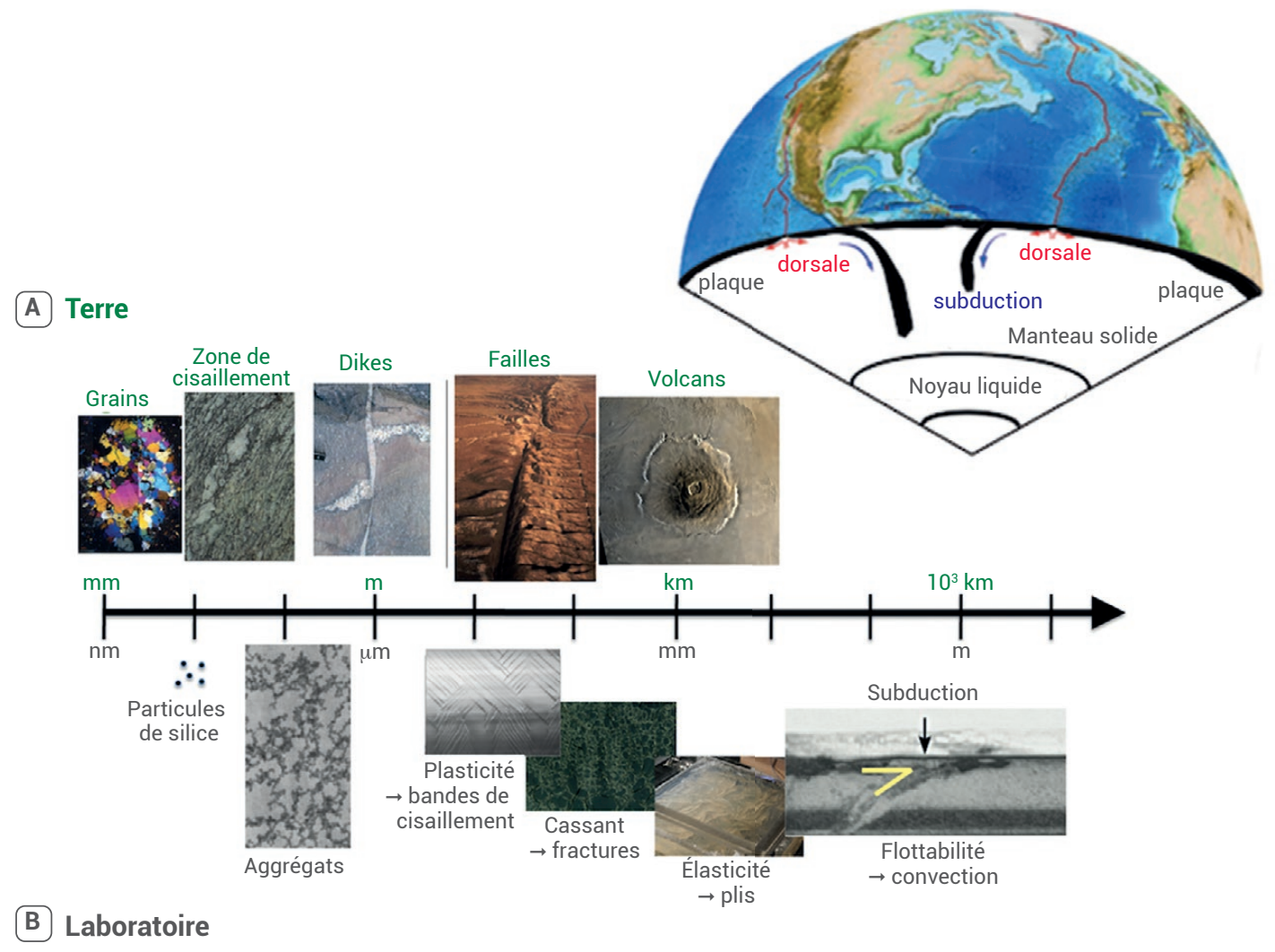

2. Les structures et phénomènes observés à différentes échelles dans des milieux hétérogènes solide-fluide soumis à un gradient de température, depuis la « brique élémentaire » contenue dans le fluide (grains pour une roche, nanoparticules pour le laboratoire), jusqu'à l'échelle de la convection. A) Sur Terre, et B) dans le laboratoire, en utilisant une dispersion aqueuse de nanoparticules de silice (le Ludox).

$>>$

de densité est égal à $\rho \alpha \Delta \mathrm{T}$, où $\alpha$ désigne le coefficient d'expansion thermique et $\rho$ la densité de référence. La convection se produit lorsque $R a$ est supérieur à une valeur critique $R a_{\mathrm{c}} \sim 1000$. $R a$ varie entre $10^{6}$ et $10^{9}$ pour les manteaux planétaires, et entre $10^{4}$ et $10^{11}$ pour les dispersions colloïdales en laboratoire. De telles valeurs impliquent la formation de "panaches ", instabilités dont la forme rappelle celle d'un champignon (fig. 1c), et qui apparaissent de manière chaotique au niveau des couches limites chaude et froide du système. Quand la viscosité dépend fortement de la température ou de la concentration en particules, le régime convectif dépend aussi du rapport maximum des viscosités $\gamma=\eta$ (surface) / $\eta$ (bas) présent dans le système. Lorsque $\gamma \geq 10^{4}$, la convection se développe sous un " couvercle stagnant", formant une «peau » ou "plaque » localisée à la surface et qui supporte la majorité des variations de viscosité. Ce couvercle, dont l'épaisseur peut devenir comparable à celle de la couche limite froide pour de grands rapports $\gamma$, ne participe pas aux mouvements de convection et limite donc fortement les transferts de chaleur et de matière vers la surface. Ceci rend la structure de la convection très asymétrique (fig. 1).

Étant donnés les grands contrastes de température à travers les manteaux planétaires $(>3000 \mathrm{~K}), \gamma>10^{10}$, et ceux-ci devraient toujours être en régime de couvercle stagnant, c'est-à-dire avec une plaque beaucoup plus visqueuse en surface mais qui ne casse et ne replonge jamais. Ceci explique bien la présence de plaques en surface sur Terre, mais pas leur fracturation, ni la subduction ou la tectonique des plaques.

En revanche, si la peau de surface a aussi des propriétés solides (plastique, élastique, cassante), on observe le développement de bandes de cisaillement, de plis et même de fractures (fig. 2A), comme dans le Ludox (fig. 2B). Cette texturation de la peau à méso-échelle modifie sa rhéologie effective et son interaction avec la convection : la plaque de surface peut alors se fracturer, dès que les contraintes dues à la formation de plis ou à la convection dépassent une valeur critique (contrainte-seuil).

\section{La subduction induite par les panaches}

Nous avons pu observer en laboratoire, dans des dispersions colloïdales soumises à un gradient de température, un nouveau mécanisme : la subduction des plaques induite par les panaches chauds et montants (fig. 3) [2]. En effet, la montée d'un panache chaud, et donc plus léger, va induire un bombement (" corona ") de la plaque en surface et la mettre en tension (fig. 3e1). Pour un panache suffisamment gros et léger, la contrainte produite peut devenir supérieure à la contrainte-seuil de rupture de la plaque, et cette dernière se fracture (fig. 3e2). Nous avons observé entre deux (fig. 3c) et cinq branches de fracture initialement. Comme le matériau du panache est plus léger que la plaque, il remonte à la surface par l'intermédiaire des fractures (fig. 3e3). Ceci induit la subduction de morceaux de plaque qui s'enfoncent sous la conjonction de leur propre poids et du poids du panache qui s'étale (fig. 3e4). La géométrie d'un panache étant axisymétrique, son étalement 

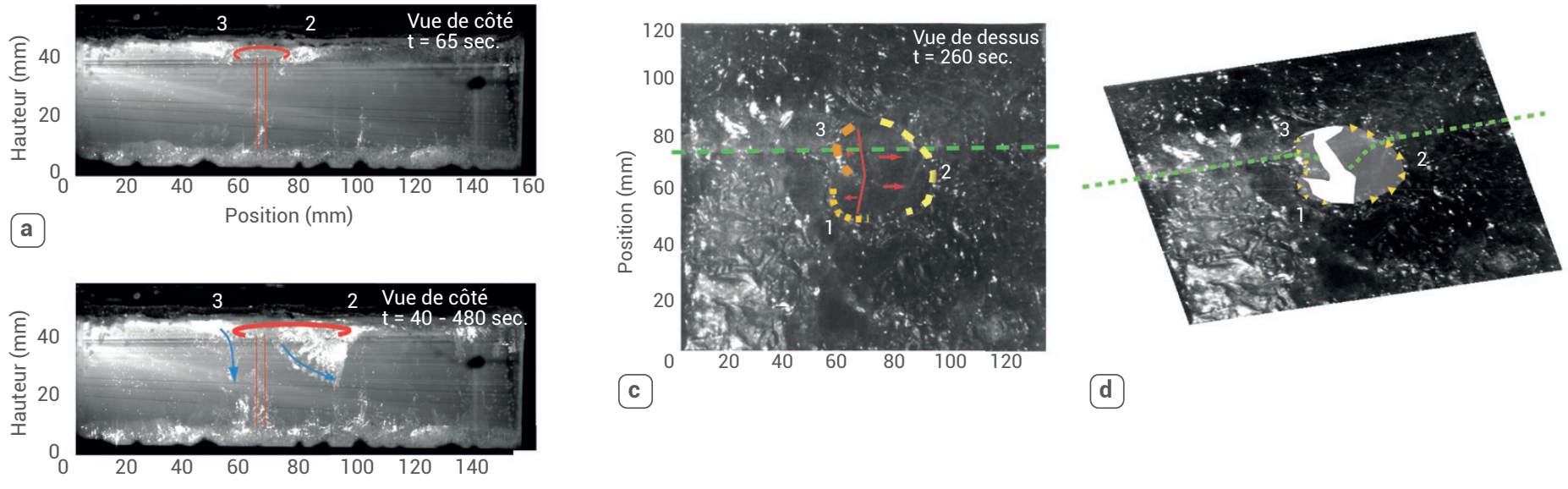

(b)
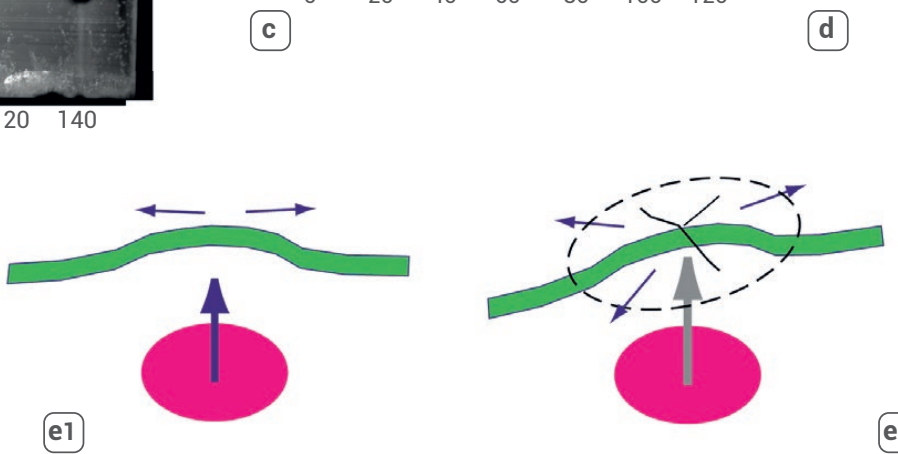

e1

e2
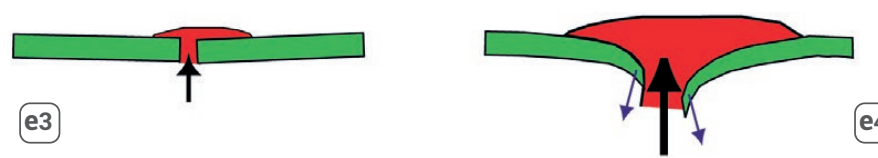

3. Subduction induite par un panache montant chaud dans une dispersion colloïdale soumise à un gradient de température.

(a) Vue de côté d'une section verticale de la cuve illuminée par une nappe laser au temps $\mathrm{t}=65 \mathrm{~s}$ après l'impact du panache sous la peau de surface. Cette dernière apparait plus brillante. On note aussi la présence de fragments de peau au fond de la cuve, ce qui est dû à des épisodes antérieurs de subduction.

(b) Superposition des images prises toutes les $10 \mathrm{~s}$ entre $\mathrm{t}=40$ et $\mathrm{t}=480 \mathrm{~s}$, montrant la subduction de deux plaques de part et d'autre du panache, et le recul au cours du temps de celle de droite.

(c) La surface de la cuve vue du dessus au temps $t=260 \mathrm{~s}$. En rouge, la trace initiale de la déchirure lors de l'impact du panache, et en jaune les trois arcs (numérotés) de subduction des plaques à $\mathrm{t}=260 \mathrm{~s}$. Les tirets verts matérialisent l'intersection avec les coupes verticales (a) et (b).

(d) Vue en trois dimensions de c), où l'intérieur de la corona a été vidé pour visualiser la subduction des trois plaques.

(e) Schémas résumant les différentes étapes de la subduction induite par le panache.

est plutôt circulaire (fig. 3c) et son empreinte à la surface s'élargit au cours du temps, ce qui pousse les subductions à reculer (fig. 3b). Si le matériau de la plaque était purement visqueux, on aurait un anneau continu de subduction qui pourrait se déformer continument pour s'élargir au cours du temps. Mais le caractère cassant de la plaque interdit cet étirement continu. C'est pourquoi la subduction ne peut se produire que le long de segments d'arc, et que la subduction d'une portion de plaque implique la présence de déchirures actives aux deux extrémités du segment. De plus, la subduction ne peut rester active et continuer de reculer que si la fracture se poursuit aux extrémités du segment. Continuer de déchirer la plaque en surface nécessitant de l'énergie, la subduction s'arrête en général lorsque le panache cesse de s'étaler. Ce processus occasionne ainsi un renouvellement local et épisodique de la surface.

L'initiation de la subduction par un panache convectif nécessite trois conditions : (i) La plaque en surface doit être intrinsèquement plus dense que le manteau ; cette condition est toujours remplie pour le Ludox. Dans le cas d'un manteau planétaire, la plaque est constituée pour sa majorité de manteau froid, mais est revêtue de la « croute " qui correspond au résidu plus léger du manteau qui a partiellement fondu en remontant vers la surface. Sur Terre, la croute océanique fait typiquement $6 \mathrm{~km}$ d'épaisseur pour une plaque épaisse d'environ $100 \mathrm{~km}$ : la plaque océanique sera donc assez dense pour subducter. En revanche, la croute continentale peut atteindre $50 \mathrm{~km}$ d'épaisseur et être encore plus légère que la croute océanique : ceci rend les continents plus légers que le manteau et empêche leur subduction.

(ii) La contrainte exercée par le panache chaud doit excéder la contrainte-seuil de la plaque. L'estimation de cette contrainteseuil est délicate car c'est une propriété effective qui varie avec le mode de déformation (de la fracture cassante au seuil de plasticité), et donc avec l'épaisseur et la structure thermique de la plaque. 
$>>$

(iii) La plaque doit pouvoir se déformer (se courber) pour accommoder la subduction. Cette dernière condition va dépendre de l'élasticité de la plaque (et donc de sa structure thermique et/ou chimique), de son poids, de la rhéologie du matériau et de l'intensité du panache.

Ces trois conditions permettent de déterminer des lois d'échelle testées sur les mesures du laboratoire. Puis les paramètres des différentes planètes sont injectés dans ces lois d'échelle pour prédire le domaine d'existence de la subduction induite par les panaches (fig. 4). Ainsi, la condition (ii) implique que seuls les panaches chauds et légers, et d'un diamètre supérieur à $500 \mathrm{~km}$, pourraient fracturer la plaque, si cette dernière n'est pas trop épaisse et trop froide (grande contrainte-seuil). D'autre part, si la plaque est trop peu dense/épaisse ou trop molle, les conditions (i)-(iii) ne seront pas remplies non plus. Les quelques analyses des roches à la surface de Vénus fournies par les sondes Venera dans les années 1970 suggèrent que les températures actuelles de l'intérieur des manteaux de Vénus et de la Terre sont comparables. En revanche, la température de surface est beaucoup plus chaude $\left(\sim 460^{\circ} \mathrm{C}\right)$ sur Vénus que sur Terre $\left(\sim 0^{\circ} \mathrm{C}\right)$. Ceci va modifier la structure en température de la plaque supérieure, et donc aussi sa densité et sa rhéologie (voir la figure 1). La figure 4 montre alors que l'initiation de la subduction par un panache est beaucoup plus favorable sur Vénus que sur la Terre.

Ceci correspond bien aux observations :

- La signature caractéristique des zones de subduction dans la topographie des fonds sous-marins sur Terre comporte une fosse de grande profondeur en arc de cercle et le bombement élastique de la plaque qui plonge dans le manteau (comme dans l'exemple donné dans la figure $5 c$ ). C'est aussi ce que nous observons dans le Ludox, encore qu'il soit difficile d'avoir des données systématiques de topographie de la surface compte tenu des contraintes expérimentales. Sur Vénus, les seules signatures de subduction repérées sur les données de la sonde Magellan se trouvent autour de grands objets circulaires appelés « coronae " (fig. 5a). Les observations géophysiques suggèrent que les grandes coronae seraient dues à l'impact de panaches chauds remontant dans le manteau vénusien. Sandwell et Schubert [3] avaient

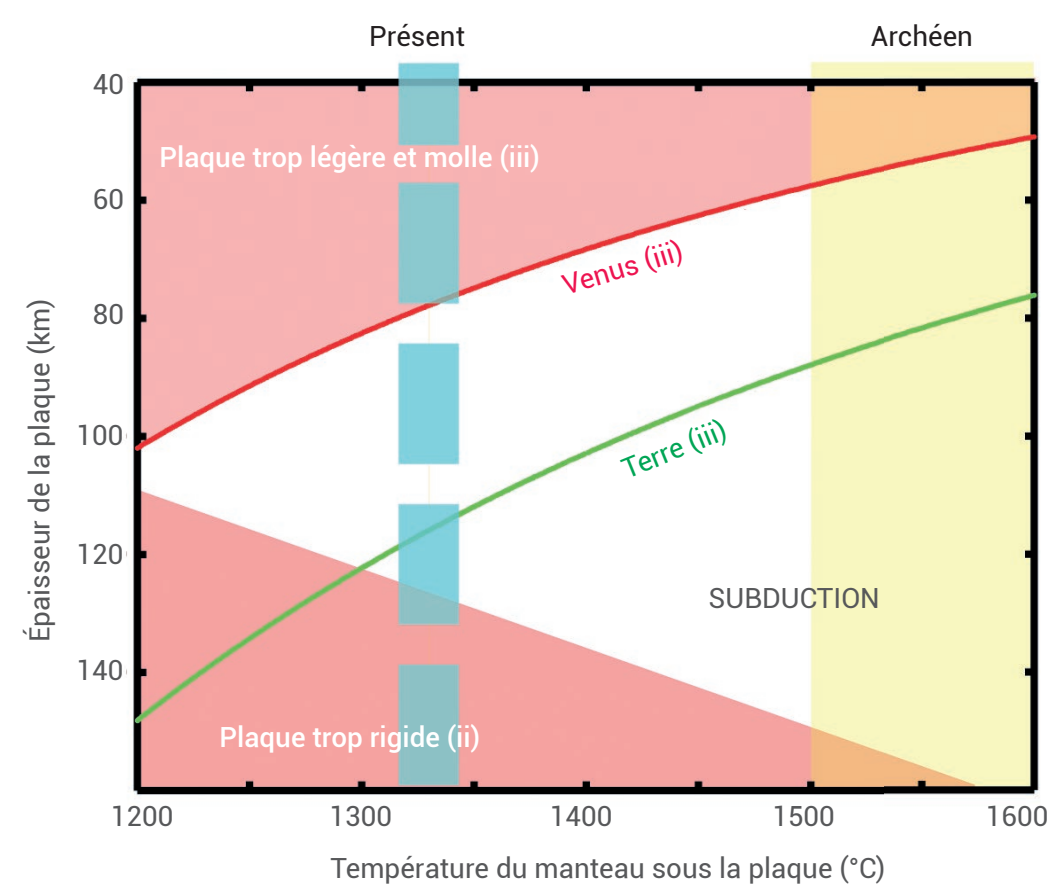

4. Domaine d'existence de la subduction induite par un panache, déduit de la modélisation, en fonction de la température du manteau juste sous la plaque et de l'épaisseur de la plaque. Le panache est $300^{\circ} \mathrm{C}$ plus chaud que la couche supérieure du manteau et $100 \mathrm{~kg} / \mathrm{m}^{3} \mathrm{moins}$ dense que la moyenne de la plaque. Dans les zones rosées, l'initiation de la subduction par les panaches est impossible car les conditions (ii) ou (iii) ne sont pas remplies. La condition (iii) est représentée en vert pour la Terre et en rouge pour Vénus. La ligne de rectangles bleus indique la gamme de températures actuelle pour les manteaux de la Terre et de Vénus. La zone jaune délimite les températures plus chaudes à l'intérieur de la Terre à l'époque archéenne (vieille de 3 à 4 milliards d'années).

proposé que ces panaches pouvaient induire la subduction, mais cette hypothèse était restée à l'état de schéma depuis. Nos expériences de laboratoire sur les dispersions colloïdales montrent que ce mécanisme est en effet physiquement et quantitativement réalisable. Une analyse plus fine des données de Magellan (radar, topographie et gravimétrie), là où la résolution est suffisante, confirme l'existence de l'association panache-subduction sur la plus grande corona, Artemis (2300 km de diamètre), et sur Quetzalpetlatl (800 km de diamètre). Dans les deux cas, la subduction avérée ne décrit pas un cercle complet mais seulement un arc (fig. 5a,b). De plus, des émissivités élevées ont été mesurées sur Quetzalpetlatl [4], et interprétées comme signature d'un volcanisme récent. Ceci suggère que le panache sous Quetzalpetlatl est bien encore chaud et actif, ce qui implique que la subduction autour de celui-ci doit aussi être active actuellement. Plus grande et sans doute à un stade plus tardif de son évolution, Artemis présente aussi à l'intérieur de la corona une grande structure ressemblant à une dorsale océanique, là où de la nouvelle plaque est créée grâce aux remontées de magma chaud. Donc, loin d'être en sommeil entre deux évènements catastrophiques, Vénus présente actuellement des activités volcanique et tectonique bien développées [5].

- Sur la Terre actuelle, seul le plateau des Caraïbes serait né de l'impact d'un panache et est depuis entouré de zones de subduction (fig. 2A). En revanche, pour un manteau terrestre plus chaud, comme il y a plus de trois milliards d'années à l'Archéen, ce mécanisme de subduction induite par des panaches devient aussi favorable que sur Vénus (fig. 4). Les embryons des continents pourraient avoir été créés de la sorte. 


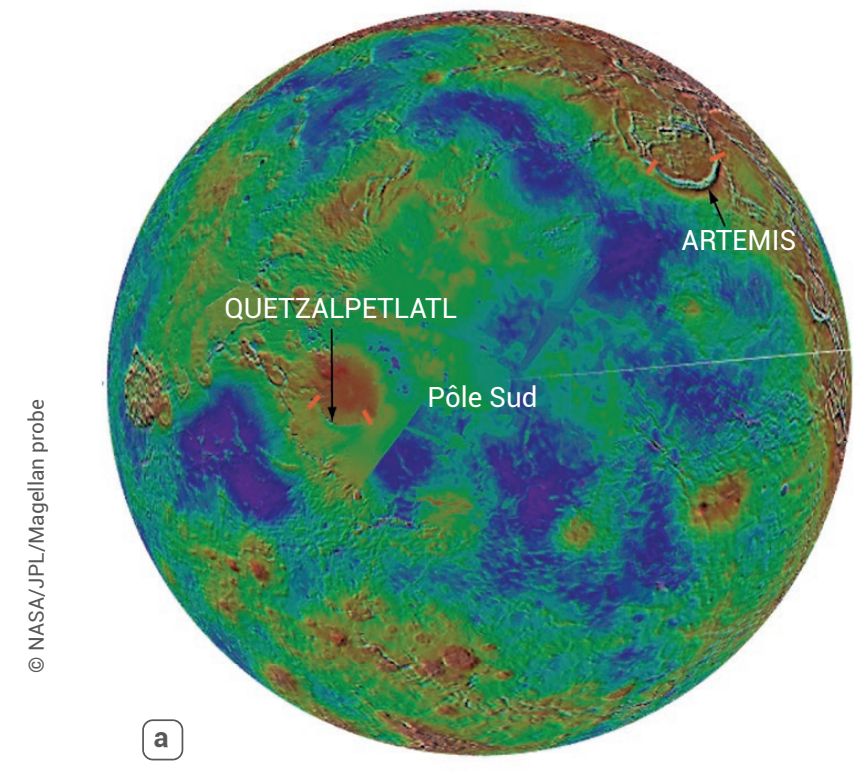

5. Structures géologiques à la surface de la planète Vénus.

(a) Image radar en fausses couleurs de la topographie de Vénus, révélée par une vue hémisphérique centrée sur le Pôle Sud prise par le satellite Magellan. Les zones élevées sont en rouge, celles de basse altitude en bleu. Des arcs de fosses de subduction ont été identifiés autour des coronae Artemis et Quetzalpetlal. Ces dernières correspondent à des bombements de la surface, dus à la poussée des panaches mantelliques chauds qui remontent.

(b) Zoom sur Artemis, la plus grande corona de Vénus. Sur les figures a et b, les tirets rouges marquent les extrémités des arcs de subduction.

(c) Profil topographique le long du segment BB' de b). La fosse de subduction et le bombement élastique de la plaque plongeante apparaissent distinctement. Au fur et à mesure que la corona s'agrandit, fosse et bombement devraient se déplacer vers B'.
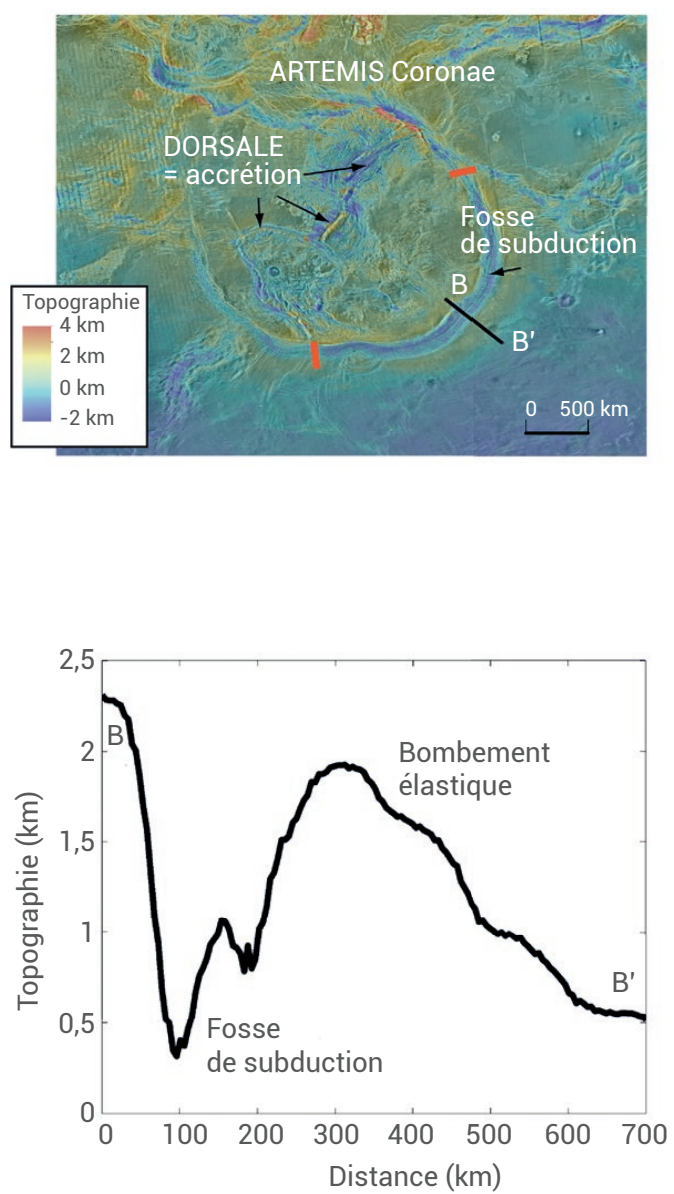

c

\section{Conclusion}

La combinaison des observations sur Vénus et de nos expériences sur les solutions colloïdales suggère l'existence d'un nouveau régime de convection, où le renouvellement de la surface n'est ni absent (comme le couvercle stagnant), ni complètement continu (comme la tectonique des plaques), ni catastrophique (comme cela avait été suggéré pour Vénus), mais se produit de façon localisée dans l'espace et le temps, au fur et à mesure que les panaches chauds arrivent à la surface.

De nombreuses questions restent encore en suspens :

- Quelles sont les conditions pour que ce régime effectue une transition vers la tectonique des plaques observée sur Terre? - Existe-t-il encore d'autres régimes convectifs ? Nous n'avons pas fini d'explorer tout l'espace des paramètres de nos expériences de matière molle.

- Il est pour l'instant impossible de quantifier l'étendue et l'intensité exactes de l'activité de Vénus, faute d'une couverture homogène et bien résolue de sa topographie et de sa gravimétrie. C'est l'objectif de l'orbiteur de la mission VERITAS, proposée à la NASA dans le cadre de l'appel Discovery. Quant à la mission EnVision, proposée à l'ESA, elle permettrait d'étudier à plus grande résolution un certain nombre de sites ciblés [6].

\section{Références}

1 P. Bacchin et al., "Drying colloidal systems: Laboratory models for a wide range of applications", Eur. Phys. J. E 41 (2018) 94.

2• A. Davaille, S.E. Smrekar et S. Tomlinson "Experimental and observational evidence for plume-induced subduction on Venus", Nature Geosciences, 10 (2017) 349-355.

3- D.T. Sandwell et G. Schubert, "Evidence for retrograde lithospheric subduction on Venus", Science 257 (1992) 766-770.

4. S.E. Smrekar et al., "Recent Hotspot Volcanism on Venus from VIRTIS emissivity data", Science 328 (2010) 605-608.

5. S.E. Smrekar, A. Davaille et C. Sotin, "Venus Interior Structure and Dynamics", Space Sci. Rev., 214 (2018) 88, doi: 10.1007/s11214-018-0518-1.

6. S. Hall, "The race to Venus", Nature, $\mathbf{5 7 0}$ (6 juin 2019)

7• M. D. Dyar, S. E. Smrekar et S. R. Kane, «Vénus : pourquoi est-elle si différente de la Terre ? ", Pour la Science 498 (avril 2019). 